\title{
Health information about chemotherapy side-effects for patients with leukemia at an oncology ward: Do nurses still lead at the frontline?
}

\author{
Amboto Justina $\mathrm{N}^{1}$, Amakali Kristofina*2 \\ ${ }^{1}$ School of Nursing; Faculty of Health Science, University of Namibia, Namibia \\ ${ }^{2}$ Department of General Nursing Science, School of Nursing, Windhoek, Namibia
}

Received: March 30, 2017

DOI: $10.5430 /$ cns.v5n3p19
Accepted: May 9, 2017

Online Published: June 6, 2017

\begin{abstract}
Chemotherapy is the universal treatment for leukemia, a common type of cancer that involves abnormal production of immature leucocytes (white blood cells). As with any medicine, chemotherapy is also associated with the side-effects. The knowledge about the side-effects of chemotherapy helps patients to better manage them, adapt to chemotherapy and thereby enhances treatment outcomes. Therefore, a descriptive cross sectional study was conducted at the Windhoek Central Hospital oncology ward to assess and describe the knowledge about side-effects of chemotherapy among adult male and female patients with leukemia and to determine if nurses provide health education to patients. The data were collected from 21 participants representing a $91 \%$ response rate of the study population. The findings indicated that the most known side-effects were the loss of hair (48\%) and vomiting (38\%). Although a significant percentage (62\%) of patients knew about the side-effects of chemotherapy, $60 \%$ of the patients received their information from the physicians. The study therefore concluded that nurses at oncology wards seem not being proactive to provide health information to patients. Recommendations were made for the nurses to increase their participation in providing health education to patients.
\end{abstract}

Key Words: Chemotherapy, Side-effects, Patients, Knowledge, Attitude

\section{INTRODUCTION}

Worldwide, more than 12 million people are newly diagnosed with cancer annually and $65 \%$ of cancer related deaths in 2012 occurs in developing countries. ${ }^{[1]}$ Concurrently, the report from the Cancer Association of Namibia as quoted in the daily local newspaper indicated that about 3,000 patients were diagnosed with cancer. ${ }^{[2]}$ Leukemia is a blood cancer characterized by the production of excessive and immature, thus ineffective white blood cells that suppress the hemopoetic and immune system. The disease is one of the top ten ranked common types of cancer. Chemotherapy is a treatment with cytotoxic medicines that kill cancer cells. Leukemia is one of the common types of cancer in Namibia and the profiles of oncology patients who are on chemotherapy is significantly increasing at the only Windhoek-based oncology hospital in Namibia. As elsewhere,$^{[3-5]}$ chemotherapy is the only feasible treatment for leukemia in Namibia. To date, little is known about local patients' knowledge of the side-effects of chemotherapy. It is therefore important to assess the knowledge about chemotherapy induced side-effects

\footnotetext{
*Correspondence: Amakali Kristofina; Email: kamakali@ unam.na; Address: Department of General Nursing Science, School of Nursing, Windhoek,
} Namibia.

Published by Sciedu Press 
among oncology patients in Namibia to identify knowledge gap. Knowledge of chemotherapy induced side-effects is important for the patient to adapt to the treatment.

Despite the fact that chemotherapy helps kill cancer cells and increases survival rates, it is also associated with many undesirable short- and long term side-effects. The short term side-effects include nausea, vomiting, headache, fatigue anxiety, depression, edema of the limbs and mouth ulcers, while long-term side-effects include susceptibility to infection, infertility, and loss of hair to mention but a few. ${ }^{[6,7]}$

However, the literature argues that although chemotherapy started as early as the 1940's, until today, patients have little knowledge about its side-effects. ${ }^{[3]}$ Therefore, patient advocates argue that a lack of proper knowledge of the side-effects becomes a challenge among leukemia patients, because the patients cannot identify alternatives to overcome the sideeffects. Rather, they experiences fear and anxiety, which indicate poor coping with the illness. ${ }^{[8]}$

The findings on the impact of education to parents of children with leukemia indicates improved quality of life for these children after the parents were provided with information on how to provide care to their children concerned. ${ }^{\text {[9] }}$ Therefore, the literature concludes that the patients should be provided with health education about their illnesses because possession of information helps patients to self-manage their condition and improve adherence to treatment. ${ }^{[8]}$ Concurrently, the literatures stress that there are better treatment outcomes from patients who are knowledgeable about chemotherapy than those who are not knowledgeable. ${ }^{[8,10-12]}$

However, failure of the health personnel to give proper health education to the patients on what they can expect causes lack of necessary information about chemotherapy side-effects among the oncology patients. ${ }^{[3]}$ There are guidelines and regulations stipulated in the handbook of the revised chemotherapy protocols ${ }^{[13]}$ that are to be followed when prescribing and administering chemotherapy to a patient of which giving a thorough explanation of the expected outcomes and the side-effects of the cytotoxic drugs is included. Despite the claim by the literature ${ }^{[1,8]}$ that the patients' knowledge of the side-effects assists them to cope, to date, there is no research based-evidence on whether patients on chemotherapy at Windhoek Central Hospital in Namibia have the knowledge about the side-effects of chemotherapy.

\section{AIM}

The aim and objective of the study was to assess and determine the knowledge of chemotherapy induced side-effects among adult male and female patients with leukemia and to determine whether nurses provide health education to patients about chemotherapy induced side-effects. The questions that arise are: Do patients with leukemia who receive chemotherapy at Windhoek Central Hospital oncology ward have the knowledge of chemotherapy side-effects? Do nurse provide health education regarding chemotherapy side-effects to oncology patients?

\section{Methodology}

\subsection{Research design}

A descriptive and cross sectional design was conducted during September, 2016 to determine and describe ${ }^{[14]}$ the oncology patients' knowledge of chemotherapy side-effects.

\subsection{Study population}

The study and target population for the study was all $(n=23)$ adult male and female patients over the age of 18 who were suffering from leukemia and receiving chemotherapy at the Windhoek Central Hospital Oncology word at the time of the study.

\subsection{Inclusion and exclusion criteria}

All adult male and female patients with leukemia who were admitted at the oncology department of Windhoek Central Hospital and who were on chemotherapy at the time of the study and who were willing to participate were eligible to participate in the study. The patients with leukemia who were under the age of 18 were excluded from the study because they would need an informed consent from their parents to participate in the study.

\subsection{Sampling and sample sizes}

The study proposed to calculate the sample size from the study population considering the $95 \%$ confidence intervals as illustrated by the formula below followed by convenient sampling method of the participants (see Equation 1).

$$
n=\frac{N}{1+N \times a^{2}}=\frac{23}{1+23 \times 0.05^{2}}=\frac{23}{1+23 \times 0.0025}=\frac{23}{1+0.0575}=21.745
$$

$n=22$

Note. $n=$ sample size, $N=$ total population, and $a=$ confidence limit $5 \%$ or $0.05 \%$ 
However, due to the small number of patients and the subsequent sample size, the entire study population $(n=23)$ was included in the study to ensure validity of the data. Out of the total number $(n=23)$ of adult male and female patients with leukemia, two of the patients declined to participate in the study and only 21 patients participated in the study. Then a convenient sampling technique was applied and a respondent rate of $91.3 \%$ was achieved.

\subsection{Data collection tool}

The data were collected through a structured questionnaire of two sections. The first section was about the demographic data of the study participants. The second section of the questionnaire was a checklist of the common chemotherapy induced side-effects that patients may experience, sources of health information and the remedial actions for counteracting the side-effects. ${ }^{[8,9]}$ The questionnaire was piloted for reliability on 2 leukemia patients who received chemotherapy at Windhoek Central Hospital, but who did not participate in the actual study.

\subsection{Data collection methods and data analysis}

The data were collected through the interview of the participants by the prime researcher. Completed questionnaires were numbered and sealed for validation by the researchers before analysis. The Statistic Package for Social Science (SPSS) was used to analyze the data and descriptive statistics analyses were performed.

\subsection{Ethical considerations}

Permission to conduct research among the patients at the health facility was obtained from the Ethical and Research Committee at the Ministry of Health and Social Services (MOHSS). Verbal permission to conduct the study within the Oncology department of the Hospital was granted by the Head of Medical Oncology Cancer Care at Oncology department. Ethical principle of justice, autonomy, respect for person and beneficence were applied to the study context as explained in the next sessions.

Principle of justice was applied by conducting the study among adult patients who received chemotherapy as treatment for leukemia because they were well placed to provide the necessary information which would serves as basis for the improvement of providing health information to patients regarding chemotherapy side-effects. All participants were treated equally. Autonomy of the participants was observed. Written informed consent was obtained from the participants after the purpose and objectives of the study were explained to them. Participation was voluntary. Privacy was ensured, as only the researchers had access to the information collected and information was not divulged to any health care providers without the permission of a respective participant. Anonymity and confidentiality were maintained as the participants were identified by code numbers, thus no information could be linked to an individual participant. The study is beneficial to both the health services and the patients who receive chemotherapy as the recommendations would contribute to the improvements of the provision of health information to patients.

\section{RESULTS}

In this section, the demographic information of the participants is presented firstly, followed by the presentation of the findings on the participants' knowledge of chemotherapy side-effects and the sources of health information to the participants.

\subsection{The demographic characteristics of the respondents} The findings indicated that the majority $(57 \%)(\mathrm{n}=12)$ of the respondents for this study were male. Likewise, $57 \%$ of the respondents fall in the age range of 18-40 years. Those of age 41-59 contributed to $23 \%(n=5)$ of the sample and only a mere $10 \%(\mathrm{n}=2)$ were 60 years and older. In addition to $29 \%(n=6)$ of the participants who had tertiary education, a significant $33 \%(\mathrm{n}=7)$ of them also had Grade 12 as their highest level of education, while $24 \%(n=5)$ had Grade 10 and only $14 \%(n=3)$ had primary education. Table 1 and 2 displays the demographic information of the study participants which can directly influence their knowledge and responses to side-effects of treatment.

Table 1. Gender and ages of the participants $(\mathrm{N}=21)$

\begin{tabular}{|c|c|c|c|c|c|}
\hline \multicolumn{3}{|c|}{ Gender of the participants } & \multicolumn{3}{|c|}{ Ages of the participants } \\
\hline Gender & Number & Percentage & Age range & Number & Percentages \\
\hline - Male & 12 & $57 \%$ & - $18-40$ years & 14 & $66.6 \%$ \\
\hline \multirow[t]{2}{*}{ - Female } & 9 & $43 \%$ & - 41-49 years & 5 & $23.8 \%$ \\
\hline & & & - Over 60 years & 2 & $9.5 \%$ \\
\hline Total & 21 & $100 \%$ & & 21 & $99.9 \%$ \\
\hline
\end{tabular}




\subsection{Knowledge of chemotherapy side-effects among the} participants

Several variables regarding the patients' knowledge of chemotherapy side-effects were assessed. These include the extent of provision of health education on chemotherapy side-effects, sources of information about the side-effects of chemotherapy to the respondents, timing of provision of information to the patients, patients' knowledge of the sideeffects of chemotherapy and the patients' interpretation of chemotherapy side-effects.

The extent of the provision of health education on chemotherapy side-effects was firstly assessed, the findings of which are displayed in Figure 1.

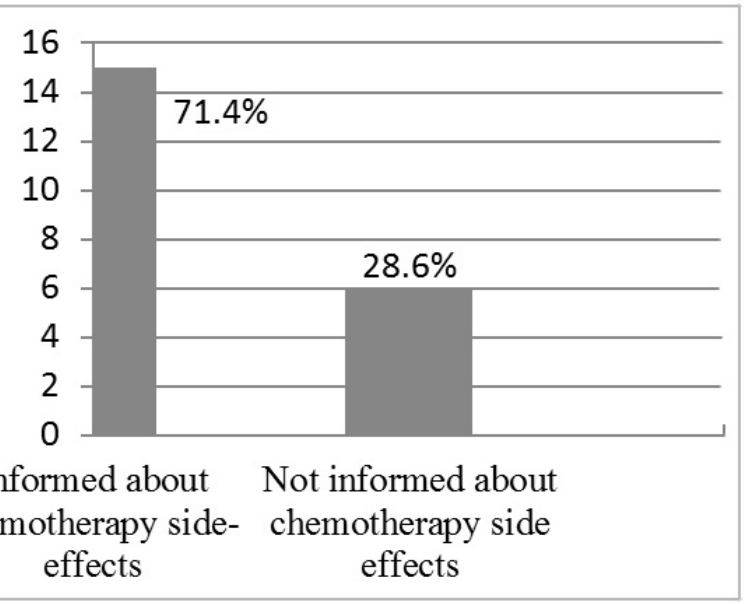

Figure 1. Number of respondents who were taught about chemotherapy side-effects $(n=15)$

According to the study, $71.4 \%$ of the participants were informed about the side-effects of chemotherapy while $28.6 \%$ were not taught about the side-effects of chemotherapy. Equally, sources of information about the side-effects of chemotherapy to the respondents were also determined as indicated in Figure 2.

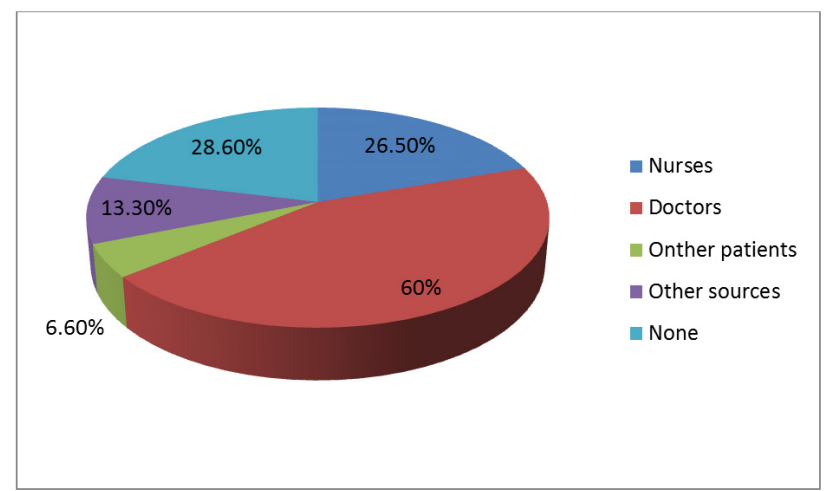

Figure 2. Sources of information about the side-effects of chemotherapy to the respondents
The findings indicated that the doctors ranked the highest $(60 \%)$ in teaching the side-effects of chemotherapy to patients as compared to a mere $26.5 \%$ of nurses who did so.

Timing of providing information to the patients was also assessed, and is displayed in the Figure 3.

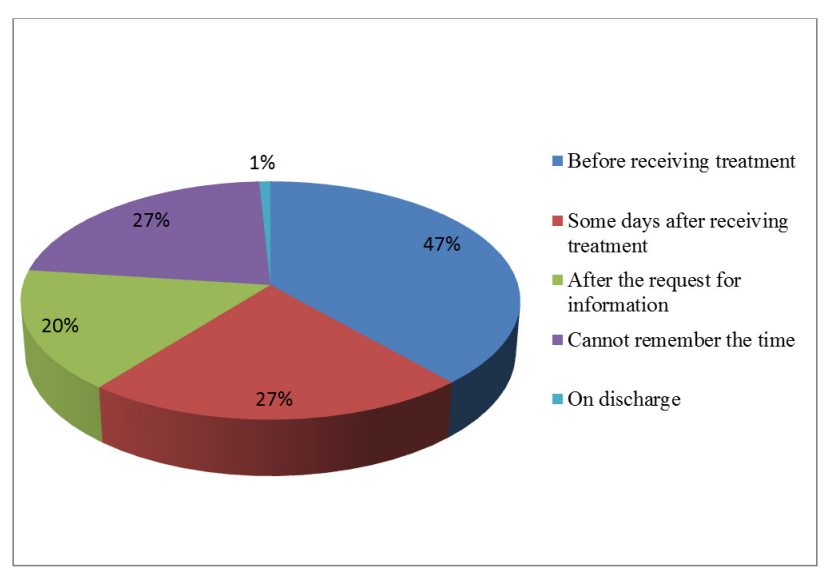

Figure 3. Period that respondents were taught about side-effects of chemotherapy

The findings indicated that $47 \%$ of the respondents were taught about the chemotherapy side-effects before receiving treatment, while $27 \%$ and $20 \%$ of the respondents were only taught about the side-effects after having received the treatment and after asking for the information respectively. Least number (1\%) of the respondents was informed about the side-effects on discharge. Furthermore, the respondents were asked to list the specific side-effects of chemotherapy that they knew. The findings are displayed in Figure 4.

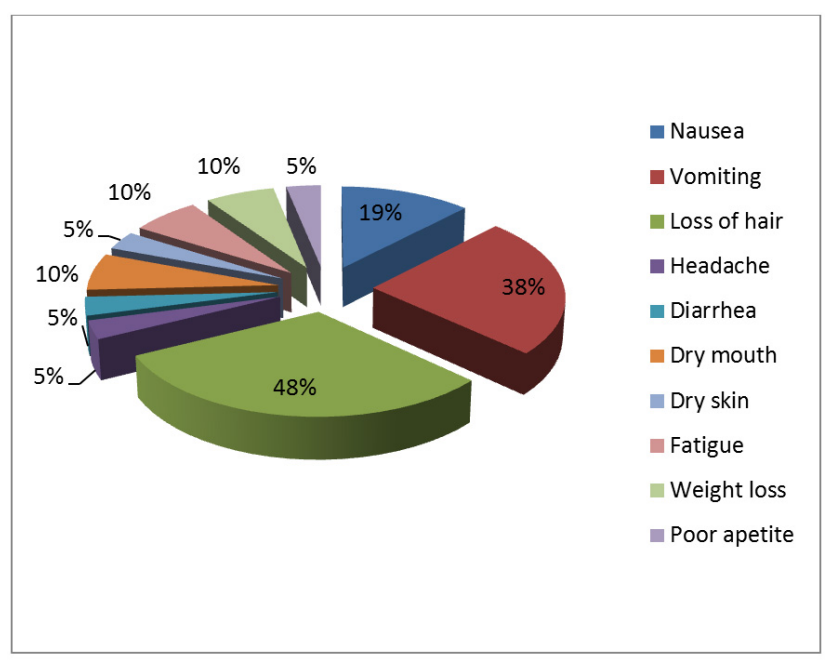

Figure 4. Knowledge of the side-effects of chemotherapy by the respondents 
The findings indicated that the most known side-effect is the loss of hair, and this is only known by 10 (48\%) of the respondents. Majority of the side-effects are only known by as few as one or two respondents.

In line with the knowledge of the side-effects of chemotherapy, the participants were asked to state the coping mechanism that they would employ to cope with the experiences of the side-effects. In that regards, the majority $48 \%$ indicated that they would do nothing about the side-effects, $43 \%$ indicated that they would try to solve it, while $1 \%$ indicates that they would ignore the side-effects.

Furthermore, the participants were asked to explain the meaning of "side-effects" and to state their opinions as to who can experience chemotherapy related side-effects. The findings indicated that $48 \%$ understood the meaning of the concept "side-effects", and equally, $48 \%$ did not know the meaning of the concept. The majority (62\%) of the participants knew that all patients on chemotherapy may experience chemotherapy related side-effect. However, $29 \%$ and $10 \%$ indicated that only older people and males respectively can experience the side-effects from chemotherapy.

\section{DisCusSiON}

The purpose of the study was to assess the knowledge about the side-effects of chemotherapy by adult patients with leukemia. Demographic information indicated that the majority representation of males in the study substantiates the fact that leukemia affects males more than females, as the literature claims that nearly three-fifths of all leukemia cases are diagnosed in men. ${ }^{[15]}$ Furthermore, the findings indicated that the cumulative majority $(86 \%)$ of the respondents were literate. By implication, they were able to apply health information if provided to them. Grasping of patients' socio-economic background would enable health care professionals to provide information that are appropriate for the patients' effective management of chemotherapy induced side-effects.

The extent of the information to patients about chemotherapy related side-effects was assessed and the findings indicated that the majority of them were informed about the side-effects of chemotherapy, with only a few who indicated not to have received the information. In comparison with suggestions by the literature which indicates that only $34 \%$ of the patients know at least one or two side-effects of chemotherapy and more than $50 \%$ of patients with leukemia do not have an idea if chemotherapy can cause any undesirable effects, ${ }^{[3]}$ the findings from this study has proven differently because the majority $(71.4 \%)$ of the respondents knew about the side-effects of their treatment.
With regard to the sources of information about chemotherapy related side-effects, medical doctors were most cited as the main sources of information to the patients, compared to nurses who spend the longest time with the patients. Surprisingly, nurses who are designated as patients' advocates scored the lowest in providing health information to the patients about the side-effects of the medications. A significant number of the participants cited other patients and printed information as the only sources of information about the side-effects from chemotherapy. The findings are in concurrence with the literature's claim that out of ten nurses, only two would go an extra mile to explain to the patients the adverse symptoms that patients may experience from the treatment. ${ }^{[16]}$ These finding are further substantiated ${ }^{[3]}$ that health care providers tend to neglect health education to patients about the side-effects of chemotherapy, however failure of which influences treatment outcome negatively.

Negligence of information to patients has been recorded as a common trend among nurses, which is presumed to be a result of perceived higher work load and demands to provide routine care to patients. ${ }^{[10,17]}$ Nevertheless, the trend compromises quality care to patients as it neglect equipping patients with the knowledge about the side-effects of treatment and the identification of alternatives to cope with the side-effects. It is therefore imperative that nurses provide health information to patients. ${ }^{[18]}$

Despite the fact that $71.4 \%$ of patients knew about the sideeffects of chemotherapy, the study showed that most of the respondents knew only one or two side-effects in spite of the vast number of the side-effects associated with chemotherapy. Majority of the side-effects are only known by as few as one or two respondents as demonstrated in Figure 4. The most known to all side-effects is loss of hair. Some side-effects such as anemia, low immunity, constipation and infertility were not listed by any of the respondents.

As regard the logistic for health education to those who received it, Figure 3 indicates that at least $47 \%$ of the respondents were taught about the side-effects of chemotherapy before receiving the treatment which is the highest percentage compared to other times when the respondents were given information about the side-effects. The findings about timing of information to patients in this study is consistent with a similar study in Malaysia, ${ }^{[1]}$ the findings of which also indicates that the majority (54\%) of the oncology patients prefer to be given information about the side-effects of chemotherapy before the initiation of chemotherapy.

However, the findings indicates that $20 \%$ and $27 \%$ of the respondents were given information on the side-effects of the medications after asking and after some days following 
the initiation of treatment respectively, while $1 \%$ were even given the information about the side-effects on discharge. Additionally, a significant $28 \%$ of the respondents expressed misconceptions that only older people and male can experience the side-effects from chemotherapy.

Patients should be given health education as per the directives of the Chemotherapy Protocols ${ }^{[13]}$ and more so, before the initiation of chemotherapy to prepare the patient on what he/she would expect and identify coping mechanisms. In that regards, the literature ${ }^{[18,19]}$ indicates that patients should be given health education in anticipation of both short termand long term chemotherapy related side-effects, with the clarification that the severity and rate of occurrence of these symptoms varies from person to person. Misconception on the predispositions to chemotherapy side-effects should be readdressed. Patients should also be granted enough time for them to ask questions and have clarity and the nurse, as the patient's advocate should ensure that this is implemented. ${ }^{[19]}$

In conclusion, health information to patients regarding the side-effects of chemotherapy creates awareness, relieve anxiety and help them to cope better with treatment related side-effects. ${ }^{[3]}$ The author further indicates that patients who are aware of potential side-effects are more likely to identify alternatives to overcome the chemotherapy related side-effects. ${ }^{[1]}$ This in return enhances positive treatment outcomes.

\section{Conclusions}

The findings of this study indicated that although oncology patients were provided with information on the side-effects of chemotherapy, a significant number demonstrated little knowledge on the variety of the side-effects of chemotherapy, while some even asserted myths about gender and age as being the predispositions to chemotherapy induced side-effects.

\subsection{Recommendations}

The findings are suggestive of the recommendations with regard to timing of providing the information to patients, improvement of quality of information to patients and the reinforcement of nurses' role in providing information to the patients. Therefore, as per the directive of the revised Protocols for Chemotherapy, all patients should be given adequate information about the side-effects of chemotherapy before the initiation of the treatment. Appropriate information should be offered to prevent misconceptions and myths around the side-effects of chemotherapy. Moreover, the role of nurses in providing health education to patients cannot be overemphasized.

\subsection{Limitations}

The study was conducted in one region of Namibia; having its own care context and problems however, these results cannot be generalized to other regions of the country, neither to other countries in the region. One more important limitation of the study is that the sample size was small, and therefore, may not be a national representative distribution of the population of all adult patients with leukemia who receive chemotherapy. Closed ended questions in the questionnaire may have limited the information from the respondents, thereby causing low validity of the data.

\section{ACKNOWLEDGEMENTS}

The researchers acknowledge the Ministry of Health and Social Services for the permission to conduct the study and collect the data among the patients at its health facilities. Special acknowledgement is hereby accorded to all oncology patients who participated in the study for their time and provision of the valuable data, which may serve as guideline for the improvement of health education by nurses to oncology patients.

\section{CONFlicts OF INTEREST Disclosure}

The authors declare they have no conflict of interest in reporting the study findings.

\section{REFERENCES}

[1] Chan HK, Ismail S. Side Effects of Chemotherapy among Cancer Patients in a Malaysian General Hospital: Experiences, Perceptions and Informational Needs from Clinical Pharmacists. Asian Pacific Journal of Cancer Prevention. 2014; 15(13): 5305-5309. https://doi.org/10.7314/APJCP. 2014.15.13.5305

[2] The Namibian Namibia: Cancer On the Rise. 3000 Namibians Diagnosed in 2013-14. Namibia: Windhoek; 2016.

[3] Ramson S. Chemotherapy Conspiracy Theories. Australia: Mapleton 2014.
[4] Intramuscular Chemotherapy. 2017. Available from: chemocare.co $\mathrm{m}$

[5] Cancer Care Nova Scotia Administration of cancer chemotherapy. 2011. Available from: www. Cancercare.n.s.ca

[6] American Cancer Society Chemotherapy side effects. 2017. Available from: www. cancer.org

[7] Healthline The side effects of chemotherapy on the body. 2017.

[8] Erickson J. Oncologic Nursing. Chicago: Rush University; 2011.

[9] Hashemi F, Asadi N, Beheshtipour N, et al. The Impact of Educating Parents of Leukemic Children on Patients' Quality of Life. 
Iranian Red Crescent Medical Journal. 2011; 13(8): 550-555. PMid: 22737526.

[10] Nader A, Hamid SN, Hadi R, et al. Nurses' attitude to patient education barriers in educational hospitals of Urmia University of Medical Sciences. Iranian Journal of Nursing \& Midwifery Research. 2012; 17(1): 12-15.

[11] Ream E, Richardson A. The role of information in patients' adaptation to chemotherapy and radiotherapy: a review of the literature. European Journal of Cancer Care. 1996; 5(3): 132. PMid: 9117045. https://doi.org/10.1111/j.1365-2354.1996.tb00223.x

[12] Douglass S. Oncology facts and theories. Cure Magazine. 2015.

[13] Chemotherapy Protocols (n.d.) 2016. Available from: http://www. indexmundi.com/nambia/chemotherapy-p rotocols.hitm/indexmundi
[14] Brink H. Fundamentals of Research Methodology for Health Professionals. Cape Town: South Africa: Lansdowne; 2006.

[15] Smeltzer SC, Bare BG, Hinkle JL, et al. Brunner and Suddarth's textbook of Medical-Surgical nursing (12th Eddition.). Philadelphia: Lippincot Williams \& Wilkins; 2010.

[16] Richardson A, Barnett W. Nursing Research in Cancer Care. London: Scutari Press; 2002.

[17] Nazarko L. Potential pitfalls in adherence to hand washing in community. British Journal of community nursing. 2009; 14(2): 64-68. PMid: 19223812.

[18] Bailey C. Cancer Nursing Changing Frontiers. London: Royal Marsden; 1992.

[19] Clarke D, Flanagan J. Advancing Nursing Practice in Cancer and Palliative Care. New York: Palgrave Macmillan; 2002. https: //doi.org/10.1007/978-1-349-88882-5 